\title{
Visual Obstruction of Flow Indicator Increases Inspiratory Volumes in Incentive Spirometry
}

\author{
Adam EM Eltorai, Thomas J Martin, Shyam A Patel, Megan Tran, Ashley S Eltorai, \\ Alan H Daniels, and Grayson L Baird
}

\begin{abstract}
BACKGROUND: Incentive spirometers were developed to facilitate sustained maximum inspiration. In addition to a slow-rising float that indicates volume displacement, the incentive spirometers includes a sensitive, rapid-fluttering flow indicator. Achieving the target inspiratory volume is believed to be the most important factor in successful incentive spirometers use. This investigation hypothesized that patients focus on the rapid fluttering of the flow indicator rather than volume float during incentive spirometers use. The effects of adjusting hand positioning to cover the flow indicator on inspiratory volumes were evaluated. METHODS: A randomized, prospective, counter-balanced crossover analysis of postoperative subjects was completed. In alternating assignment, the subjects were randomized to 1 of 2 study groups: (A) with the flow indicator covered first followed by the flow indicator standard exposed, and (B) with the flow indicator standard exposed first, followed by the flow indicator covered. The subjects were asked to perform 2 inhalations on their incentive spirometers in the first flow indicator condition: covered or the standard exposed. After a 2-min delay, the subjects were then asked to perform an additional 2 inhalations with the alternate flow indicator condition. The difference between mean inspiratory volumes under covered and standard exposed conditions was evaluated for all subjects, within and between groups. RESULTS: A total of 42 subjects were evaluated. For all the subjects, there was a mean increase of $255.4 \mathrm{~mL}$ of inspired volume when the flow indicator was covered $(1,869.0$ vs $1,613.7 \mathrm{~mL}, P<.001)$. For the subjects in group B who had their flow indicators covered after standard exposure, mean inspiratory volumes increased by $285.7 \mathrm{~mL}(1,613.1 \mathrm{vs} 1,898.8 \mathrm{~mL}, P=$ .009). For subjects in group $A$ who had their flow indicator covered before standard exposure, their mean inspiratory volumes increased by $225.0 \mathrm{~mL}(1,614.3 \mathrm{vs} 1839.3 \mathrm{~mL}, P=.007)$ when covered. There was no significant difference between the mean increases across patients in groups $A$ and $B$ (225.0 vs $285.7 \mathrm{~mL}, P=.63$ ). CONCLUSIONS: Covering the flow indicator during incentive spirometers significantly increased achieved inspiratory volumes. Increased volumes were generated, irrespective of flow indicator covering order, which strongly suggested that the covering effect was greater than any learning or condition order carry-over effects. Because achieving target inspiratory volumes is considered the most important factor in successful incentive spirometers use, these findings may have immediate applications for improving incentive spirometers protocols, patient education, and device design implications. Key words: incentive spirometry; quality improvement; flow; inspiratory volume; respiratory care; hand positioning; patient education; human factors. [Respir Care 2019;64(5):590-594. ( 2019 Daedalus Enterprises]
\end{abstract}

\section{Introduction}

Ubiquitously prescribed ${ }^{1}$ to reduce postoperative pulmonary complications, incentive spirometers were origi-

Dr AEM Eltorai, Mr Martin, Dr Patel, Ms Tran, Dr Daniels, and Dr Baird are affiliated with the Warren Alpert Medical School of Brown University, Providence, Rhode Island. Dr AS Eltorai is affiliated with Yale nally developed to facilitate sustained maximum inspiration. ${ }^{2}$ Achieving target inspiratory volume is believed to be the most important factor in successful incentive spi-

University School of Medicine, New Haven, Connecticut. Dr AEM Eltorai and Mr Martin are co-first authors.

Disclosures: No funding was received for this work. 
rometers use. ${ }^{3}$ Furthermore, as an objective marker of deepbreathing effort, inspiratory volume is a commonly used outcome measure, ${ }^{4-9}$ which has been correlated with various clinical outcomes. ${ }^{10-12}$ Unfortunately, many patients may not know how to optimally use incentive spirometers and maximize inspiratory volume in the postoperative setting. ${ }^{13}$

Difficulties with incentive spirometers use may stem, in part, from the device design. In addition to a slow-rising float that indicates volume displacement (Fig. 1C), the incentive spirometers includes a sensitive, rapid-fluttering flow indicator (Fig. 1A). The flow indicator includes symbols with instructional suggestions for patients about maintaining adequate flow (Fig. 1A). Further, the motion onset of the rapid-fluttering flow indicator occurs before that of the volume float. It is well-established that attention is drawn to moving objects in the visual field. ${ }^{14-18}$ In particular, motion onset (the state of no motion changing to some motion) ${ }^{16}$ has been demonstrated to draw attention, which is needed to effectively perform tasks. ${ }^{19-21}$ With the incentive spirometers flow indicator, such cues may draw the patients' attention toward the flow indicator rather than toward the volume. In so doing, patients may fail to focus on maximizing inspiratory volume. Hence, the objective of the present investigation was to evaluate the effects of covering the flow indicator with hand positioning to determine the corresponding effects on inspiratory volumes. It was hypothesized that covering the flow indicator during patient use would increase inspiratory volume.

\section{Methods}

\section{Study Design}

This investigation was a single-center, randomized experiment. Postoperative patients on the orthopedic surgery service at a large academic medical center were evaluated; all the patients were prescribed an incentive spirometers as standard of care and were thus eligible for inclusion. This study was approved by our medical center's institutional review board (45 CFR $\S 46.110(5)$ ). Over $2 \mathrm{~d}$ in November and December 2017, data were collected from consenting subjects. To create a counter-balanced crossover

Dr AEM Eltorai discloses a relationship with Springer and Lippincott Williams \& Wilkins. Dr Daniels discloses relationships with SpineArt, Globus, Orthofix, Springer, and Stryker. The remaining authors have disclosed no conflicts of interest.

Correspondence: Adam EM Eltorai PhD, Warren Alpert Medical School of Brown University, 222 Richmond Street, Providence, RI 02906. E-mail: adam_eltorai@brown.edu.

DOI: $10.4187 /$ respcare.06331

\section{QUICK LOOK}

\section{Current knowledge}

Incentive spirometry is commonly used by patients in the postoperative period to help promote maximal inspiration and prevent pulmonary complications.

\section{What this paper contributes to our knowledge}

Patients perform larger inhalations using IS when the flow meter is visually obstructed. These findings suggest a redesign of IS may be useful.

design, a simple alternating assignment was used to randomize subjects to 1 of 2 study groups based on successive patient room numbers.

\section{Study Groups}

Subjects randomized to study group A attempted IS by initially having their flow indicator covered (Fig. 2A). This was then followed by repeating the attempt with the flow indicator standard exposed (Fig. 2B). The subjects randomized to group B had this order reversed to attempt incentive spirometers with their flow indicator standard initially exposed, which was then followed by a repeated attempt with the flow indicator covered. The subjects were asked to perform 2 inhalations with their incentive spirometers in the first flow indicator condition. After a 2-min delay, the subjects were then asked to perform 2 addition inhalations with the alternate flow indicator condition (Table 1). Inspiratory volume was measured by the peak height of the volume displacement float (Fig. 3). The higher of the 2 inspiratory volumes from each condition was recorded.

\section{Statistical Analysis}

The mean inspiratory volumes were calculated for all subjects during the covered $\left(\mathrm{ISV}_{\mathrm{C}}\right)$ and standard exposed $\left(\mathrm{ISV}_{\mathrm{S}}\right)$ conditions, and individual differences were evaluated $\left(\Delta \mathrm{ISV}=\mathrm{ISV}_{\mathrm{C}}-\mathrm{ISV}_{\mathrm{S}}\right)$. Paired $t$ tests were used to test differences in individual performance $\left(\mathrm{ISV}_{\mathrm{C}} \mathrm{vs} \mathrm{ISV}_{\mathrm{S}}\right)$ among all the subjects and among those in each subgroup. To assess the presence of the confounding effects between the subgroups, the mean volumes and $\Delta \mathrm{ISV}$ of subjects in groups A and B were assessed for equality by using $t$ tests.

\section{Results}

A total of 42 postoperative subjects were included, each of whom had IS prescribed after different orthopedic procedures and consented to the investigation. A total of 21 subjects 

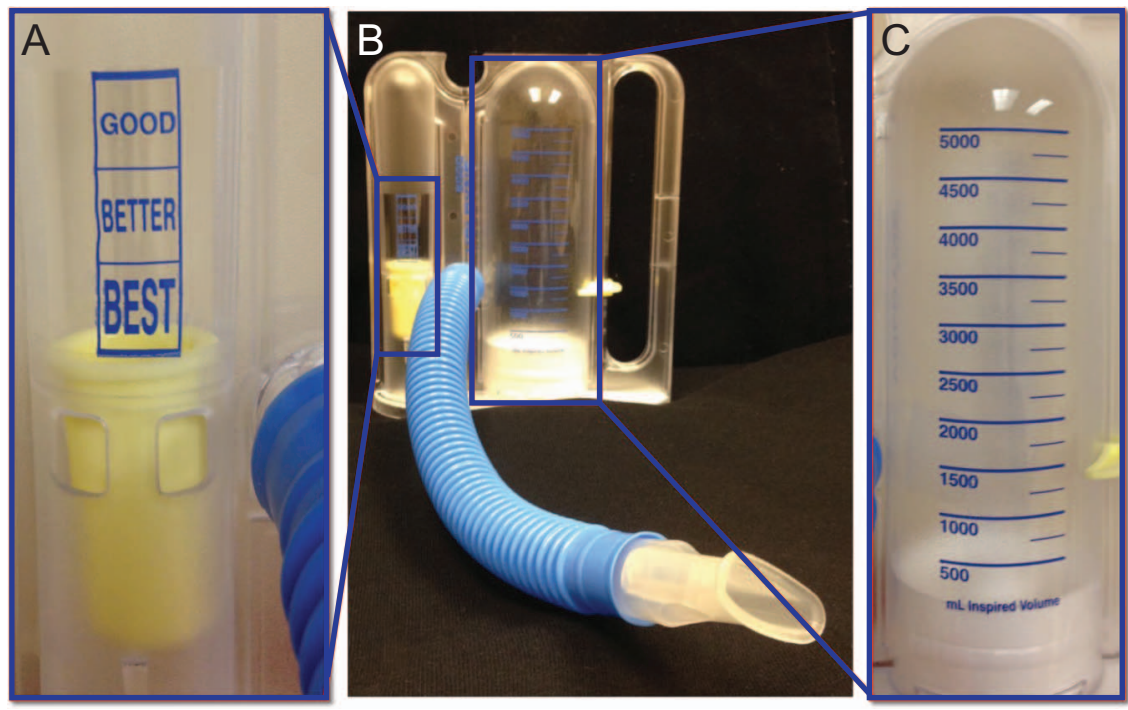

Fig. 1. A: Flow indicator close up. B: Incentive spirometry unit. C: Volume chamber close up.

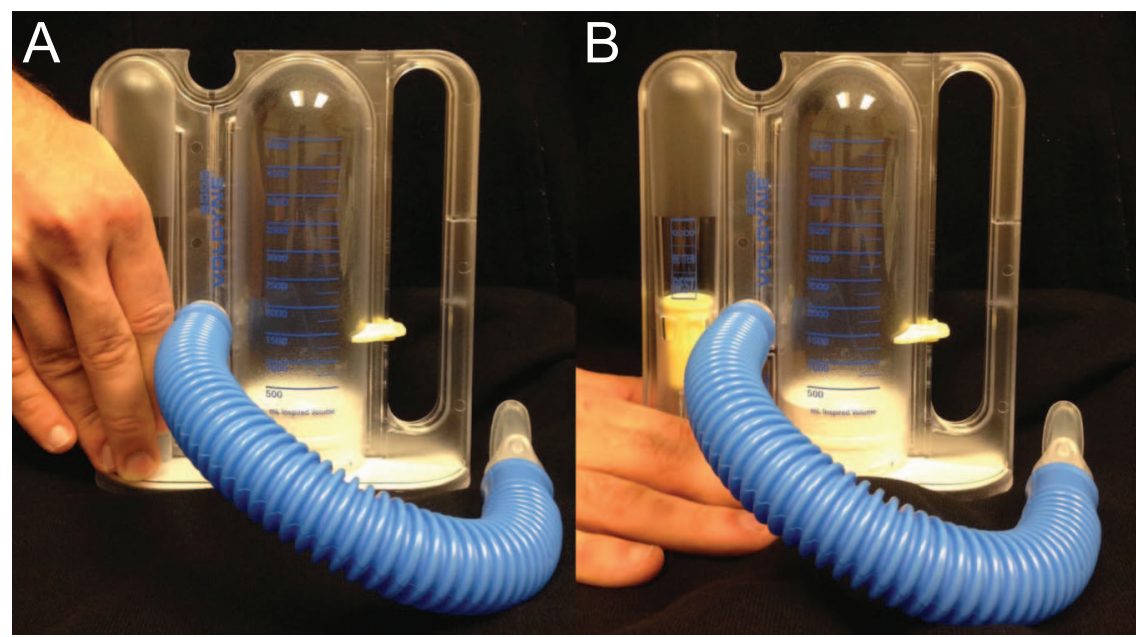

Fig. 2. Flow indicator conditions. A: Covered. B: Standard exposed.

Table 1. Diagram of Intervention by Group

\begin{tabular}{|c|c|c|c|c|c|c|c|}
\hline Group & $\begin{array}{l}\text { First Flow } \\
\text { Indicator Condition }\end{array}$ & $\begin{array}{c}\text { No. } \\
\text { Inhalations }\end{array}$ & Recorded & Delay & $\begin{array}{l}\text { Second Flow } \\
\text { Indicator Condition }\end{array}$ & $\begin{array}{c}\text { No. } \\
\text { Inhalations }\end{array}$ & Recorded \\
\hline A & Covered & 2 & Higher of the 2 inspiratory volumes & 2-min education delay & Standard exposed & 2 & Higher of the 2 inspiratory volumes \\
\hline B & Standard exposed & 2 & Higher of the 2 inspiratory volumes & 2-min education delay & Covered & 2 & Higher of the 2 inspiratory volumes \\
\hline
\end{tabular}

were randomized to study group A and 21 to study group B. By using a counter-balanced study design, the subjects served as their own controls, which avoided the need for data collection of subject demographics or characteristics.

\section{All Subjects}

Overall, the subjects performed IS with a mean (SD) inspiratory volume of 1,613.7 (695.9) $\mathrm{mL}$ and 1,869.0 (755.5) $\mathrm{mL}$ with the flow indicator exposed and covered, respectively. Pairwise analysis demonstrated a significant difference in inspiratory volumes when the flow indicator was covered by hand positioning $(P<.001)$, with a mean increase of $255.4 \mathrm{~mL}$ (Table 2).

\section{Subgroup Analysis}

There were no significant differences between subject performance in groups A versus B under the standard exposed $(1,614.3$ vs $1,613.1 \mathrm{~mL}, P>.99)$ and covered con- 
ditions $(1,839.3$ vs $1,898.8 \mathrm{~mL}, P=.80)$. In each group, there were significant increases between inspiratory volumes during the covered condition: group A $\mathrm{ISV}_{\mathrm{S}}$ of $1,614.3 \mathrm{~mL}$ versus group $\mathrm{A} \mathrm{ISV}_{\mathrm{C}}$ of $1839.3 \mathrm{~mL}(P=$ 0.0068); and group B ISV $\mathrm{S}_{\mathrm{S}}$ of 1,613.1 $\mathrm{mL}$ versus group B $\mathrm{ISV}_{\mathrm{C}}$ of $1,898.8 \mathrm{~mL}(P=.009)$. The subjects in groups $\mathrm{A}$ and $\mathrm{B}$ had mean increases of 225.0 and $285.7 \mathrm{~mL}$, respectively, under the covered condition. However, there was no statistically significant difference in performance improvement between the groups (225.0 vs $285.7 \mathrm{~mL}$, $P=.63)$. Of note, subjects in group A performed IS under the covered condition first; thus, the $\Delta \mathrm{ISV}$ in this subgroup represented a $225.0 \mathrm{~mL}$ decrease in inspiratory volume when incentive spirometers was repeated with standard exposure. Although the subjects in both groups showed improved inspiratory volume when the flow indicator was covered, there was no statistically significant difference in mean $\triangle \mathrm{ISV}$ of group A versus group B (225.0 vs $285.7 \mathrm{~mL}$, $P=.63)$.

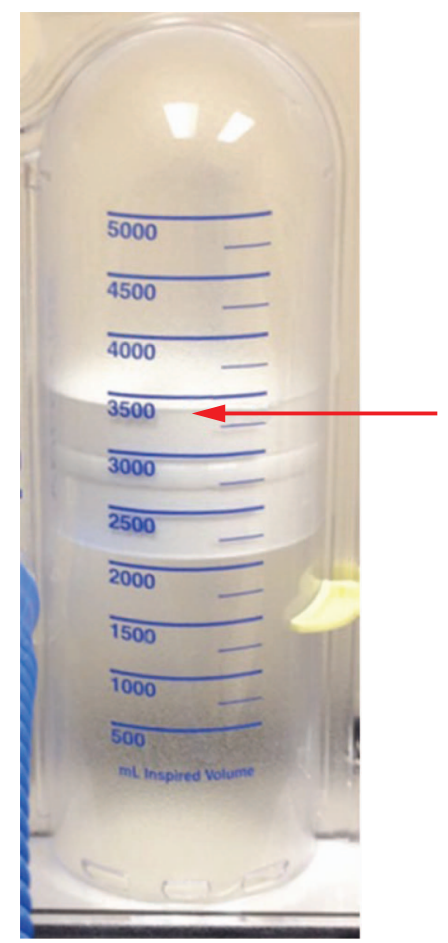

Fig. 3. Inspiratory volume measurement (red arrow).

\section{Discussion}

A prospective, counter-balanced crossover analysis of postoperative subjects who served as their own controls was completed to evaluate the effects of covering the flow indicator on inspiratory volumes in incentive spirometers. As was hypothesized, covering the flow indicator during use by the subject increased inspiratory volumes. Increased volumes were generated irrespective of flow indicator covering order, which strongly indicated that the covering effect was greater than any learning or condition order carry-over effects. A recent national survey demonstrated that most nurses and respiratory therapists consider achieving target inspiratory volume to be the most important factor in successful incentive spirometers. ${ }^{3}$ In addition, inspiratory volume during incentive spirometers has repeatedly been identified as a surrogate for inspiratory capacity ${ }^{4-9}$ and is correlated with clinical outcomes. ${ }^{10-12}$

To our knowledge, this was the first investigation to evaluate covering the flow indicator in incentive spirometers. The increase in inspiratory volumes during the covered versus standard exposed condition indicated that previous incentive spirometers studies may not have captured accurate maximum inspiratory volumes. Moreover, this was the first investigation to evaluate how incentive spirometers was administered and to highlight its importance.

The study was potentially limited by the relatively small sample of subjects included in this analysis. Future studies may benefit from larger samples and evaluation of subjects with a variety of conditions, such as those with rib fractures, those who require intensive care, or those who have undergone cardiothoracic or upper abdominal surgery. Because this study was at a single center, a multicenter investigation could strengthen the findings and make the results more generalizable. By design, subject demographics and baseline characteristics were not collected because the subjects served as their own control. Therefore, there was no need to perform cohort matching. Further, there were no significant differences between baseline inspiratory performances in each subgroup. Data collection was completed on randomly selected dates and times. Interesting further investigations may include the

Table 2. Impact of Covering Flow Indicator on Inspiratory Volumes

\begin{tabular}{|c|c|c|c|c|}
\hline \multirow{2}{*}{ Group } & \multicolumn{2}{|c|}{$\begin{array}{l}\text { Inspiratory Volume, } \\
\text { mean } \pm \mathrm{SD} \mathrm{mL}\end{array}$} & \multirow{2}{*}{$\begin{array}{l}\Delta \text { Inspiratory Volume, } \\
\text { mean }(95 \% \mathrm{CI}) \mathrm{mL}\end{array}$} & \multirow{2}{*}{$P$, paired } \\
\hline & Standard Exposed & Covered & & \\
\hline A: covered first $(n=21)$ & $1,614.3 \pm 770.9$ & $1,839.3 \pm 754.3$ & $225.0(69.5-380.5)$ & .007 \\
\hline B: standard exposed first $(n=21)$ & $1,613.1 \pm 631.1$ & $1,898.8 \pm 774.2$ & $285.7(80.4-491.0)$ & .009 \\
\hline A and B (all patients) $(N=42)$ & $1,613.7 \pm 695.9$ & $1,869.0 \pm 755.5$ & $255.4(131.9-378.8)$ & $<.001$ \\
\hline
\end{tabular}


following: various subject samples, a control for postoperative day, subject eye gaze patterns, subject distraction during incentive spirometers, impact of greater inspiratory volumes on clinical outcomes, comparison of different incentive spirometers devices, and the interaction of anesthesia type on cognition and incentive spirometers compliance.

\section{Conclusions}

Human factors, for example, hand placement during incentive spirometers performance, may affect successful utilization and overall clinical effectiveness of incentive spirometers use. Because achieving target inspiratory volumes is considered the most important factor in successful incentive spirometers use, these findings may have immediately applicable implications for optimizing incentive spirometers protocols, device design, and patient education.

\section{REFERENCES}

1. O'Donohue WJ. National Survey of the Usage of Lung Expansion Modalities for the Prevention and Treatment of Postoperative Atelectasis Following Abdominal and Thoracic Surgery. Chest 1985; 87(1):76-80.

2. Bartlett RH, Krop P, Hanson EL, Moore FD. Physiology of yawning and its application to postoperative care. Surg Forum 1970;21:222224.

3. Eltorai AEM, Baird GL, Eltorai AS, Pangborn J, Antoci V Jr, Cullen HA, et al. Perspectives on incentive spirometry utility and patient protocols. Respir Care 2018;63(5):519-531.

4. Edelen C, Perlow M. A comparison of the effectiveness of an opioid analgesic and a nonpharmacologic intervention to improve incentive spirometry volumes. Pain Manag Nurs 2002;3(1):36-42.

5. Pieracci FM, Lin Y, Rodil M, Synder M, Herbert B, Tran DK, et al. A prospective, controlled clinical evaluation of surgical stabilization of severe rib fractures. J Trauma Acute Care Surg 2016;80(2):187194.

6. Baker WL, Lamb VJ, Marini JJ. Breath-stacking increases the depth and duration of chest expansion by incentive spirometry. Am Rev Respir Dis 1990;141(2):343-346.
7. Dias CM, Vieira Rde O, Oliveira JF, Lopes AJ, Menezes SL, Guimarães FS. Three physiotherapy protocols: effects on pulmonary volumes after cardiac surgery. J Bras Pneumol 2011;37(1):54-60.

8. Cattano D, Altamirano A, Vannucci A, Melnikov V, Cone C, Hagberg CA. Preoperative use of incentive spirometry does not affect postoperative lung function in bariatric surgery. Transl Res 2010; 156(5):265-272.

9. Harton SC, Grap MJ, Savage L, Elswick RK. Frequency and predictors of return to incentive spirometry volume baseline after cardiac surgery. Prog Cardiovasc Nurs 2007;22(1):7-12.

10. Butts CA, Brady JJ III, Wilhelm S, Castor L, Sherwood A, McCall A, et al. Do simple beside lung function tests predict morbidity after rib fractures? Am J Surg 2017;213(3):473-477.

11. Harris DJ, Hilliard PE, Jewell ES, Brummett CM. The association between incentive spirometry performance and pain in postoperative thoracic epidural analgesia. Reg Anesth Pain Med 2015;40(3):232238.

12. Bastin R, Moraine JJ, Bardocsky G, Kahn RJ, Mélot C. Incentive spirometry performance. A reliable indicator of pulmonary function in the early postoperative period after lobectomy? Chest 1997;111(3): 559-563.

13. Martin TJ, Patel SA, Tran M, Eltorai AS, Daniels AH, Eltorai AEM. Patient factors associated with successful incentive spirometry. RI Med J 2018;101(9):14-18.

14. Franconeri SL, Simons DJ. Moving and looming stimuli capture attention. Percept Psychophys 2003;65(7):999-1010.

15. Franconeri SL, Simons DJ. The dynamic events that capture visual attention: A reply to Abrams and Christ. Percept Psychophys 2005; 67(6):962-966.

16. Abrams RA, Christ SE. Motion onset captures attention. Psychol Sci 2003;14(5):427-432.

17. Abrams RA, Christ SE. Onset but not offset of irrelevant motion disrupts inhibition of return. Percept Psychophys 2005;67(8):14601467.

18. Abrams RA, Christ SE. The onset of receding motion captures attention: comment on Franconeri and Simons (2003). Percept Psychophys 2005;67(2):219-223.

19. Wilimzig C, Tsuchiya N, Fahle M, Einhäuser W, Koch C. Spatial attention increases performance but not subjective confidence in a discrimination task. J Vis 2008;8(5):7.1-10

20. Cohen MR, Maunsell JH. Attention improves performance primarily by reducing interneuronal correlations. Nat Neurosci 2009;12(12): 1594-1600.

21. Yeshurun Y, Carrasco M. Spatial attention improves performance in spatial resolution tasks. Vision Res 1999;39(2):293-306. 